\title{
SCALARIZATION AND CONVERGENCE IN UNIFIED SET OPTIMIZATION
}

\author{
Khushboo $^{1, *}$ And C.S. LAlitha ${ }^{2} \odot$
}

\begin{abstract}
This paper deals with scalarization and stability aspects for a unified set optimization problem. We provide characterization for a unified preference relation and the corresponding unified minimal solution in terms of a generalized oriented distance function of the sup-inf type. We establish continuity of a function associated with the generalized oriented distance function and provide an existence result for the unified minimal solution. We establish Painlevé-Kuratowski convergence of minimal solutions of a family of scalar problems to the minimal solutions of the unified set optimization problem.
\end{abstract}

Mathematics Subject Classification. 54C60, 90C26, 49J53.

Received June 18, 2021. Accepted November 16, 2021.

\section{INTRODUCTION}

Set optimization has attracted a huge attention in last few decades due to numerous applications in variety of fields, such as economics, finance, control theory, game theory, statistics and engineering, for details see [22] and references therein.

Scalarization is one of the widely used techniques to study vector and set optimization problems in terms of associated scalar problems. In this technique, minimal solutions of the given problem are usually characterized in terms of minimal solutions of the associated parametric scalar problems. The scalarization technique includes linear and nonlinear scalarization approaches; see $[2,3,22,31]$. The most commonly used nonlinear scalarizing functions are Gerstewitz function [8] and oriented distance function [14] as they have nonconvex separation properties as the key feature. One of the important features of the oriented distance function is that it is appropriate even when the ordering set has empty interior.

Various extensions of oriented distance function in the study of set optimization have been considered in literature $[4,10,17-19,23,35]$. Ha [10] introduced one such extension to define a Hausdorff-type distance, which was later used by Chen et al. [4] and Jiménez et al. [18] to derive scalar characterizations of certain set order relations with respect to cone and the corresponding notions of minimal solutions. Characterizations of set relations with respect to cone through various extensions of the oriented distance have been investigated in $[15,17,18]$.

Keywords. Unified set optimization, nonlinear scalarization, oriented distance function, semicontinuity, Painlevé-Kuratowski convergence.

1 Department of Mathematics, University of Delhi, Delhi 110007, India.

2 Department of Mathematics, University of Delhi South Campus, Benito Juarez Road, New Delhi 110021, India.

*Corresponding author: thakurkhushboo4@gmail.com 
Set optimization problem involves comparison of sets by means of set relations. A variety of set relations with respect to convex cones and their applications in certain areas have been studied in the literature $[22,23,25]$. Attempts have been made in the past to study optimization problems in a unified setting by means of general preference relations [6,7,24,32,34]. For vector optimization problems, Rubinov and Gasimov [34] introduced a unified preference relation based on a conic set. Flores-Bazán and Hernández [6] and Flores-Bazán et al. [7] introduced a unified notion of minimality with respect to a preference relation induced by a nonempty proper set which was further extended to study scalarization scheme for set optimization problems in [24].

Apart from determining solutions of vector and set optimization problems, scalarization techniques have been used to study stability aspects as well. Stability theory deals with the investigation of the behaviour of solution sets of the perturbed problems where perturbations are on the feasible set, objective function or ordering set. One of the stability aspects studied in the literature is in terms of convergence of solution sets (see [9,21]). In order to study stability via scalarization the associated scalarized problem is perturbed instead of the original problem and then the convergence of solution sets of perturbed scalarized problems to the solution set of the given problem is established. In vector case, a lot of work has been done in this direction (see [20,30] and references therein). Moreover, in case of set optimization, the study of stability on the ground of scalarization techniques is not much advanced. Recently, Liu et al. [33] introduced monotonicity properties of set-valued map via scalarizations and investigated the stability aspect in terms of continuity.

Continuity and convexity of the scalarizing functions play an important role in establishing existence and stability results. Han and Huang [12] established the continuity and convexity of a generalized Gerstewitz function, whereas Kuwano and Tanaka [28] established the continuity of a set-valued map using the continuity of the nonlinear scalarizing function considered in [27]. Recently, Huerga et al. [15] investigated the continuity and convexity of six types of scalarizing functions based on the oriented distance function. Scalarization techniques have been used to study properties of continuity of set-valued maps in [16, 29].

In this paper, we study scalaraization using oriented distance function and stability results for a unified set optimization problem. We define a unified notion of minimal solutions with respect to a preference set relation considered in [24]. Using a generalized oriented distance function of sup-inf type, which extends a scalarizing function considered in [15], we establish scalar characterizations of preference set relation and minimal solutions. The set of minimal solutions is characterized as the union of minimal solutions of a family of parametric scalar problems where the objective function is given in terms of the generalized oriented distance function. We also investigate the continuity of a map associated with the generalized oriented distance function which leads to the existence result of unified minimal solutions as well as for the minimal solutions considered in [24]. We also observe that the existence result in this paper is different from the one established in [24]. The complete characterization of minimal solutions and the continuity of the associated map are further used to study stability results in terms of convergence of solution sets. We establish the lower and upper convergence of the minimal solution sets of a sequence of perturbed scalarized problems, obtained by perturbing the feasible set, to the set of minimal solutions of the unified set optimization problem in Painlevé-Kuratowski sense. Although, the notion considered in this paper is stronger than the notion of minimal solution considered in [24], it enables us to study scalarizations and convergence aspects of some well-known notions of minimal solution sets which can not be determined by means of scalarization established in [24]. Also, by means of examples we demonstrate that the scalarization results established in this paper extend the scalarization results of [18] with alternative assumptions.

The organization of the paper is as follows. In Section 2, we recall some basic notions and results to be used in the sequel. In Section 3, we introduce a unified notion of minimal solutions and study characterizations of preference set relation and minimal solutions in terms of generalized oriented distance function. We also deal with complete scalarizations for minimal solution sets in terms of minimal solutions of a family of parametric scalar problems. Section 4 deals with the continuity of the function associated with oriented distance function and Section 5 deals with the lower and upper convergence of minimal solution sets in Painlevé-Kuratowski sense. Section 6 provides some concluding remarks. 


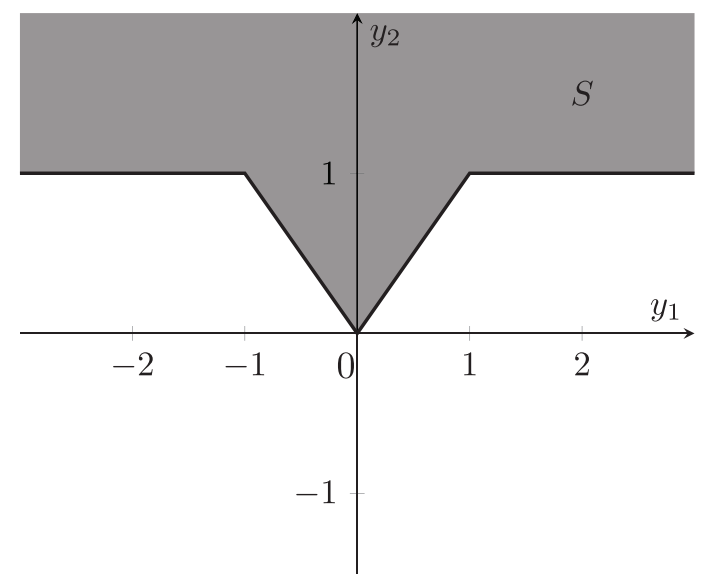

FiguRE 1. Set inducing quasi order relation.

\section{PreliminaRies}

Let $Y$ be a real normed linear space and $\mathcal{P}(Y)$ denote the set of all nonempty subsets of $Y$. For a set $A \in \mathcal{P}(Y)$ we denote the interior, closure, boundary and complement of $A$ by $\operatorname{int} A, \operatorname{cl} A, \partial A$ and $A^{c}$, respectively.

From [24] we consider the lower set preference relation $\preceq_{S}^{l}$ in $\mathcal{P}(Y)$ induced by a nonempty proper subset $S$ of $Y$. For $A, B \in \mathcal{P}(Y)$

$$
A \preceq_{S}^{l} B \Longleftrightarrow B \subseteq A-S .
$$

This preference relation unifies some of the set relations studied in literature (see [24], Rem. 3.1). Also, it can be seen that this preference relation is reflexive if $0_{Y} \in S$ and transitive if $S+S \subseteq S$. Preference relation induced by a convex cone is a quasi order relation, that is, it is both reflexive and transitive. Observe that the preference relation induced by $S=\mathbb{N} \cup\{0\}$ in $\mathcal{P}(\mathbb{R})$, where $\mathbb{N}$ is the set of natural numbers, is a quasi order relation. Another example of a quasi order relation in $\mathcal{P}\left(\mathbb{R}^{2}\right)$ is the one induced by $S=\left\{\left(y_{1}, y_{2}\right) \in \mathbb{R}^{2}: y_{2} \geq\right.$ $\left.\left|y_{1}\right|\right\} \cup\left\{\left(y_{1}, y_{2}\right) \in \mathbb{R}^{2}: y_{2} \geq 1\right\}$, as shown in Figure 1 .

Motivated by a unified solution concept considered by Flores-Bazán et al. [7], a preference relation $\preceq_{S}$ is given in [24] for elements of $Y$. For $a, b \in Y$,

$$
a \preceq_{S} b \Longleftrightarrow a-b \in S .
$$

Clearly, the preference relation $\preceq_{S}^{l}$ in $\mathcal{P}(Y)$ is an extension of the preference relation $\preceq_{S}$ in $Y$. Also, we recall from [24] that for a set $A \in \mathcal{P}(Y)$ an element $\bar{a} \in A$ is said to be an $S$-minimal point of $A$ if there does not exist any $a \in A \backslash\{\bar{a}\}$, such that $a \preceq_{S} \bar{a}$. We denote the set of $S$-minimal points of $A$ by $E_{S}(A)$.

We now recall continuity notions for a set-valued map from Khan et al. [22]. For convenience, we propose to use the term semicontinuity in place of continuity.

Definition 2.1. Let $X$ and $Y$ be real normed linear spaces and $\bar{x} \in X$. A set-valued map $F: X \rightrightarrows Y$ is said to be

(i) ([22], Def. 3.1.1(a)) upper semicontinuous at $\bar{x}$ if for any open set $V \subseteq Y$ with $F(\bar{x}) \subseteq V$ there exists a neighborhood $U$ of $\bar{x}$ such that $F(u) \subseteq V$, for all $u \in U$.

(ii) ([22], Def. 3.1.1(b)) lower semicontinuous at $\bar{x}$ if for any open set $V \subseteq Y$ with $F(\bar{x}) \cap V \neq \emptyset$ there exists a neighborhood $U$ of $\bar{x}$ such that $F(u) \cap V \neq \emptyset$, for all $u \in U$.

The set-valued map $F$ is upper (resp. lower) semicontinuous on a set $\Omega \subseteq X$ if it is upper (resp. lower) semicontinuous at every $x \in \Omega$. 
We next recall sequential characterizations of upper and lower semicontinuity of the set-valued map $F$.

Lemma $2.2([22])$. Let $X$ and $Y$ be real normed linear spaces, $F: X \rightrightarrows Y$ and $\bar{x} \in X$.

(i) ([22], Prop. 3.1.9). If $F(\bar{x})$ is compact, then $F$ is upper semicontinuous at $\bar{x}$ iff for any sequences $\left(x_{n}\right)_{n \in \mathbb{N}} \subseteq$ $X$ with $x_{n} \rightarrow \bar{x}$ and $\left(y_{n}\right)_{n \in \mathbb{N}}$ with $y_{n} \in F\left(x_{n}\right)$, there exist a subsequence $\left(y_{n_{k}}\right)_{k \in \mathbb{N}}$ of $\left(y_{n}\right)_{n \in \mathbb{N}}$ and $\bar{y} \in F(\bar{x})$ such that $y_{n_{k}} \rightarrow \bar{y}$.

(ii) ([22], Prop. 3.1.6(iv)). $F$ is lower semicontinuous at $\bar{x}$ iff for any sequence $\left(x_{n}\right)_{n \in \mathbb{N}} \subseteq X$ with $x_{n} \rightarrow \bar{x}$ and $\bar{y} \in F(\bar{x})$ there exists a sequence $\left(y_{n}\right)_{n \in \mathbb{N}}$ with $y_{n} \in F\left(x_{n}\right)$ for sufficiently large $n$ such that $y_{n} \rightarrow \bar{y}$.

The distance of a point $y \in Y$ from a set $A \subseteq Y$ is defined as $d(y, A):=\inf _{a \in A}\|y-a\|$. From [14] we recall that, the oriented distance function with respect to the set $A$ is a function $\Delta_{A}: Y \rightarrow \mathbb{R} \cup\{ \pm \infty\}$ defined as

$$
\Delta_{A}(y):=d(y, A)-d\left(y, A^{c}\right) .
$$

Clearly, $\Delta_{\operatorname{int} A}=\Delta_{A}=\Delta_{\operatorname{cl} A}$ if $A$ is convex set with nonempty interior (see [22], Page 233). Also, $\Delta_{\emptyset}(y)=+\infty$ and $\Delta_{Y}(y)=-\infty$, for all $y \in Y$.

We now state the nonconvex separation properties from Zaffaroni [36].

Proposition 2.3 ([36], Prop. 3.2). For $S \in \mathcal{P}(Y), S \neq Y$, the following assertions hold:

(i) $\Delta_{S}$ is real-valued and Lipschitzian with rank 1.

(ii) $\Delta_{S}(y)<0$ for every $y \in \operatorname{int} S, \Delta_{S}(y)=0$ for every $y \in \partial S$ and $\Delta_{S}(y)>0$ for every $y \in \operatorname{int} S^{c}$.

\section{SCAlar CHARACTERIZATIONS OF PREFERENCE RELATION AND MiNimal SOLUTIONS}

Using oriented distance function, Chen et al. [4] derived characterizations of set order relations induced by a closed convex pointed cone. In this section, we first provide scalar characterizations of the preference set relation using oriented distance function.

In the following theorem the proof of the first part follows on the lines of Proposition 3.8 in [4] and second part follows from Theorem 4.11 in [18].

Theorem 3.1. (i) If $A, B \in \mathcal{P}(Y)$, then

$$
A \preceq_{S}^{l} B \Rightarrow \sup _{b \in B} \inf _{a \in A} \Delta_{S}(a-b) \leq 0 .
$$

If $S$ is closed and $\inf _{a \in A} \Delta_{S}(a-b)$ is attained for each $b \in B$, then

$$
\sup _{b \in B} \inf _{a \in A} \Delta_{S}(a-b) \leq 0 \Rightarrow A \preceq{ }_{S}^{l} B .
$$

(ii) If int $S \neq \emptyset$ and $A, B \in \mathcal{P}(Y)$, then

$$
A \preceq_{\text {int } S}^{l} B \Longleftrightarrow \inf _{a \in A} \Delta_{S}(a-b)<0, \text { for each } b \in B .
$$

The following example shows that the reverse implication fails to hold in the absence of either of the assumptions of the first part.

Example 3.2. Let $Y=\mathbb{R}^{2}, A=(-1,0) \times(-1,0)$ and $B=\{(0,0)\}$. If $S$ is as in Figure 1 , then $S$ is closed but $\inf _{a \in A} \Delta_{S}(a-b)=0$ is not attained for $b=(0,0) \in B$. If we replace $A$ by $[-1,0] \times[-1,0]$ and $S$ by $\operatorname{int} S$, then $\inf _{a \in A} \Delta_{S}(a-b)=0$ is attained at $a=(0,0)$ but $S$ is not closed. In either case, we have $\sup _{b \in B} \inf _{a \in A} \Delta_{S}(a-b)=\inf _{a \in A} \Delta_{S}(a)=0$ but $B \nsubseteq A-S$. 
Remark 3.3. If $S=-K$, where $K$ is a closed convex proper pointed cone, then Theorem 3.1(i) reduces to Proposition 3.8 of [4]. Also, the same characterization has been established in Theorem 4.1 of [18] for the case when the set $S=-K, K$ being a proper convex cone, under the assumption that $A$ is $K$-closed, that is, the set $A+K$ is closed.

We now consider the following set optimization problem:

$$
\begin{gathered}
S \text {-Minimize } F(x) \\
\text { subject to } x \in M,
\end{gathered}
$$

where $F: X \rightrightarrows Y$ is a set-valued map, $X$ is a real normed linear space and $M$ is a nonempty subset of $X$.

With the help of the preference relation $\preceq_{S}^{l}$ a notion of $S$-l-minimal solution was introduced in Definition 5.1 of [24] for a set optimization problem. We recall that an element $\bar{x} \in M$ is said to be an $S$-l-minimal solution of $(\mathrm{P})$ if

$$
F(x) \preceq_{S}^{l} F(\bar{x}), x \in X \Rightarrow F(\bar{x}) \preceq_{S}^{l} F(x) .
$$

We next propose a stronger notion of minimal solutions, namely $S$-l-minimizers.

Definition 3.4. An element $\bar{x} \in M$ is said to be an $S$-l-minimizer of (P), if there does not exist any $x \in M \backslash\{\bar{x}\}$ such that $F(x) \preceq_{S}^{l} F(\bar{x})$.

Clearly, every $S$-l-minimizer is an $S$-l-minimal solution. We denote the set of $S$-l-minimizers of $(\mathrm{P})$ by $S$-l-Mzer. If the set-valued map $F: \mathbb{R} \rightrightarrows \mathbb{R}^{2}$ is defined as

$$
F(x)=[0,|x|] \times[0,|x|], \text { for all } x \in \mathbb{R},
$$

$M=[0,1]$ and $S$ is as in Figure 1, then it can be seen that $S$-l-Mzer $=\{1\}$.

Remark 3.5. The notion $S$-l-minimizer unifies several existing notions of minimal and approximate minimal solutions available in the literature. From Lemma 3.1 of [24] we observe that if $0_{Y} \in S^{c}, S+S \subseteq S$, and $E_{S}(F(u)) \neq \emptyset$ for each $u \in M$, then the notion of $S$-l-minimizer coincides with the notion of $S$-l-minimal solution. Further, if $S=-K$, where $K$ is a proper convex cone, then we have the notion of strict $l$-minimal solutions considered in Definition 5.1(iii) of [18]. Also, for $\epsilon>0$ and $e \epsilon-\operatorname{int} K$, if we choose $S=-\epsilon e-\operatorname{int} K$, then the set $S$-l-Mzer reduces to the set of approximate weak $l$-minimal solutions considered in Definition 4.1 of [5]. Moreover, for $S=-\operatorname{int} K$ if $F$ is $K$-proper, that is, $F(u)+K \neq Y$, for each $u \in M$, and $K$-closed valued, then the notion of $S$-l-minimizer reduces to the notion of weak $l$-minimal solutions considered in [37], Page 3770.

We next establish that the set of $S$-l-minimizers is closed under the upper semicontinuity assumption on $F$.

Theorem 3.6. If $S$ is open and $F$ is upper semicontinuous and compact-valued on $M$, then $S$-l-Mzer is closed.

Proof. Let $\left(x_{n}\right)_{n \in \mathbb{N}} \subseteq S$-l-Mzer be such that $x_{n} \rightarrow \bar{x}$. Suppose on the contrary $\bar{x} \notin S$-l-Mzer. Then, there exists $x \in M \backslash\{\bar{x}\}$ such that

$$
F(\bar{x}) \subseteq F(x)-S .
$$

Since $x_{n} \in S$-l-Mzer it follows that there exists $y_{n} \in F\left(x_{n}\right)$ such that

$$
y_{n} \notin F(x)-S \text {. }
$$

By Lemma 2.2(i) there exist $\bar{y} \in F(\bar{x})$ and a subsequence $\left(y_{n_{k}}\right)_{k \in \mathbb{N}}$ of $\left(y_{n}\right)_{n \in \mathbb{N}}$ such that $y_{n_{k}} \rightarrow \bar{y}$. Since $S$ is open, therefore from (3.2) we have $\bar{y} \notin F(x)-S$ which contradicts (3.1).

Remark 3.7. In view of Remark 3.5, the above theorem provides the closedness of the set of weak $l$-minimal solutions for $S=-\operatorname{int} K$. However, Han and Huang ([11], Prop. 2.3) required an additional assumption that the feasible set is closed, to establish the same result. 
The following example justifies that the above theorem may not hold in the absence of upper semicontinuity of $F$.

Example 3.8. Let $F: \mathbb{R} \rightrightarrows \mathbb{R}^{2}$ be defined as

$$
F(x)= \begin{cases}\{(0, t): 0 \leq t \leq 1\}, & \text { if } x>0 \\ \{(0,0)\}, & \text { if } x \leq 0\end{cases}
$$

$M=(-1,1)$ and $S=\left\{\left(y_{1}, y_{2}\right) \in \mathbb{R}^{2}: y_{2}>y_{1}^{2}\right\}$. Clearly, $F$ is not upper semicontinuous on $M$ and $S$-l-Mzer $=$ $(-1,0]$.

The following characterizations for $S$-l-minimizers of (P) easily follow from Theorem 3.1.

Theorem 3.9. For $\bar{x} \in M$, the following assertions hold:

(i) For each $x \in M \backslash\{\bar{x}\}$, let $\inf _{y \in F(x)} \Delta_{S}(y-\bar{y})$ be attained for every $\bar{y} \in F(\bar{x})$. If $S$ is closed, then $\bar{x}$ is an S-l-minimizer if and only if

$$
\bar{y} \in F(\bar{x}) \sup \inf _{y \in F(x)} \Delta_{S}(y-\bar{y})>0, \text { for all } x \in M \backslash\{\bar{x}\} .
$$

(ii) If $S$ is open, then $\bar{x}$ is an $S$-l-minimizer if and only if for all $x \in M \backslash\{\bar{x}\}$

$$
\inf _{y \in F(x)} \Delta_{S}(y-\bar{y}) \geq 0 \text {, for some } \bar{y} \in F(\bar{x}) .
$$

We next characterize $S$-l-minimzers in terms of minimal solutions of a family of parametric scalar problems. In this regard, we first define an oriented distance map $\mathcal{D}: \mathcal{P}(Y) \times \mathcal{P}(Y) \rightarrow \mathbb{R} \cup\{ \pm \infty\}$ as

$$
\mathcal{D}(A, B):=\sup _{b \in B} \inf _{a \in A} \Delta_{S}(a-b) .
$$

Clearly, $\mathcal{D}$ is finite-valued if the sets $A$ and $B$ are compact. The function $\mathcal{D}$ was introduced by Ha [10] in which they considered $-K$ instead of $S$ where $K$ being a proper closed convex pointed cone. This function has also been considered in $[4,15,17-19]$.

The parametric scalar minimization problem associated with $u \in M$ is

$$
\begin{array}{ll}
(\mathrm{P}(u)) \quad & \text { Minimize } \mathcal{D}(F(x), F(u)) \\
& \text { subject to } x \in M .
\end{array}
$$

We denote the set of minimal (resp. strict minimal) solutions of $(\mathrm{P}(u))$ by $\operatorname{argmin} \mathrm{P}(u)$ (resp. st $\operatorname{argmin} \mathrm{P}(u)$ ), that is,

$$
\begin{aligned}
\operatorname{argminP}(u) & =\{\bar{x} \in M: \mathcal{D}(F(\bar{x}), F(u)) \leq \mathcal{D}(F(x), F(u)), \text { for all } x \in M\} \\
\text { (resp. st } \operatorname{argminP}(u) & =\{\bar{x} \in M: \mathcal{D}(F(\bar{x}), F(u))<\mathcal{D}(F(x), F(u)), \text { for all } x \in M \backslash\{\bar{x}\}\}) .
\end{aligned}
$$

The next lemma gives a monotonic implication in terms of the scalar map $\mathcal{D}$.

Lemma 3.10. If $S+S \subseteq S$ and $A, B, C \in \mathcal{P}(Y)$, then the following assertions hold:

(i) $A \preceq_{S}^{l} B \Rightarrow \mathcal{D}(A, C) \leq \mathcal{D}(B, C)$.

(ii) If $S$ is open and, $B$ and $C$ are compact sets, then

$$
A \preceq_{S}^{l} B \Rightarrow \mathcal{D}(A, C)<\mathcal{D}(B, C) .
$$


Proof. We only prove (ii) as the proof of (i) follows on similar lines. Let $A \preceq_{S}^{l} B$ and $b \in B$. Then, there exists $a^{\prime} \in A$ such that $b-a^{\prime} \in-S$. As $S+S \subseteq S$ we have $b-S \subset a^{\prime}-S$. Clearly, for any $c \in C$ we have

$$
d\left(c, a^{\prime}-S\right) \leq d(c, b-S) \text { and } d\left(c,(b-S)^{c}\right) \leq d\left(c,\left(a^{\prime}-S\right)^{c}\right)
$$

with at least one of the inequality being strict as $S$ is open. Hence,

$$
\inf _{a \in A} \Delta_{S}(a-c) \leq \Delta_{S}\left(a^{\prime}-c\right)<\Delta_{S}(b-c) .
$$

Since $B$ is compact and the above inequality holds for each $b \in B$ we have

$$
\inf _{a \in A} \Delta_{S}(a-c)<\inf _{b \in B} \Delta_{S}(b-c)
$$

which implies that $\inf _{a \in A} \Delta_{S}(a-c)<\mathcal{D}(B, C)$. As $\Delta_{S}$ is continuous therefore the function $g(c):=\inf _{a \in A} \Delta_{S}(a-$ $c)$ is upper semicontinuous and hence, the compactness of $C$ implies that $\mathcal{D}(A, C)<\mathcal{D}(B, C)$.

Remark 3.11. The following example shows that the compactness assumption of the sets cannot be relaxed in Lemma 3.10(ii).

Example 3.12. Let $Y=\mathbb{R}^{2}$ and $S$ be the interior of the set in Figure 1 . If $A=B=(-1,0) \times(-1,0)$ and $C=\{(0,0)\}$, then $A \preceq \preceq_{S}^{l} B$ and $\mathcal{D}(A, C)=\mathcal{D}(B, C)=0$. However, if $A=[-1,0] \times[-1,0], B=\{(-1,-1)\}$ and $C=\mathbb{R}_{+}^{2}$, then $A \preceq_{S}^{l} B$ and $\mathcal{D}(A, C)=\mathcal{D}(B, C)=+\infty$.

The following lemma will be used in the sequel.

Lemma 3.13. For $A \in \mathcal{P}(Y)$, if $0_{Y} \in \operatorname{cl} S$ and $E_{S}(A) \neq \emptyset$, then $\mathcal{D}(A, A)=0$.

Proof. Let $\bar{a} \in A$, hence $\inf _{a \in A} \Delta_{S}(a-\bar{a}) \leq \Delta_{S}\left(0_{Y}\right)$. As $0_{Y} \in \operatorname{cl} S$ we obtain $\inf _{a \in A} \Delta_{S}(a-\bar{a}) \leq 0$ which further implies that $\mathcal{D}(A, A) \leq 0$.

Let $\hat{a} \in E_{S}(A)$, then $a-\hat{a} \in S^{c}$ for every $a \in A \backslash\{\hat{a}\}$. This implies that $\inf _{a \in A} \Delta_{S}(a-\hat{a})=0$ as $0_{Y} \in \operatorname{cl} S$ and thus $\mathcal{D}(A, A)=\sup _{\bar{a} \in A} \inf _{a \in A} \Delta_{S}(a-\bar{a}) \geq \inf _{a \in A} \Delta_{S}(a-\hat{a})=0$.

The following example shows that the lemma may fail to hold in the absence of either of the assumptions.

Example 3.14. Let $Y=\mathbb{R}^{2}$ and $A=\{(y, 0): 0<y<1\}$. If $S=\left\{\left(y_{1}, y_{2}\right) \in \mathbb{R}^{2}: y_{2} \geq 1\right\}$, then $0_{Y} \notin \operatorname{cl} S$. However, $E_{S}(A)=A$ and $\mathcal{D}(A, A)=1$. If $S=\left\{\left(y_{1}, y_{2}\right) \in \mathbb{R}^{2}: y_{2} \geq-1\right\}$, then $0_{Y} \in \operatorname{cl} S$ but $E_{S}(A)=\emptyset$ and $\mathcal{D}(A, A)=-1$.

Remark 3.15. (i) Ha ([10], Lem. 3.4(ii)) proved that $\mathcal{D}(A, A)=0$, for $S=-K$ where $K$ is a closed convex pointed cone, provided either int $K=\emptyset$ or $E_{-K}(A) \neq \emptyset$.

(ii) Assuming $K$ to be a proper convex cone, Jiménez et al. ([18], Prop. 3.15) proved that $\mathcal{D}(A, A)=0$, if $A$ is $K$-proper, that is, $A+K \neq Y$. Now, if we consider $A=\left\{\left(y_{1}, y_{2}\right) \in \mathbb{R}^{2}: 0 \leq y_{1} \leq 1\right\} \backslash\left\{\left(y_{1}, y_{2}\right) \in \mathbb{R}^{2}: 0<\right.$ $\left.y_{1} \leq 1, y_{2}=0\right\}$ and $K=\left\{\left(y_{1}, y_{2}\right) \in \mathbb{R}^{2}: y_{2}=0\right\}$, then $A$ is not $K$-proper whereas $E_{(-K)}(A)=\{(0,0)\}$ is nonempty. Evidently, $\mathcal{D}(A, A)=0$.

(iii) It may further be noted that Proposition 3.15 of [18] cannot be extended assuming $A$ to be $S$-proper, that is, $A-S \neq Y$ and thus results of [18] cannot be extended for arbitrary set. For instance in Example 3.14, for $S=\left\{\left(y_{1}, y_{2}\right) \in \mathbb{R}^{2}: y_{2} \geq-1\right\}$, the set $A-S \neq Y$ but $\mathcal{D}(A, A)=-1$.

In the next theorem, we characterize an $S$-l-minimizer of $(\mathrm{P})$ in terms of a strict minimal (resp. minimal) solution of a parametric scalar problem provided $S$ is a closed (resp. open) set in $\mathcal{P}(Y)$. As a consequence we obtain complete characterizations of the set of $S$-l-minimizers of (P). Using Theorem 3.9, Lemmas 3.10 and 3.13, the proof of the first and the second part of the following theorem easily follows on the lines of Theorems 5.6 and 5.7 in [18], respectively. 
Theorem 3.16. Let $\bar{x} \in M, 0_{Y} \in \operatorname{cl} S$ and $E_{S}(F(\bar{x})) \neq \emptyset$. Then, the following assertions hold:

(i) For each $x \in M \backslash\{\bar{x}\}$, if $\inf _{y \in F(x)} \Delta_{S}(y-\bar{y})$ is attained for every $\bar{y} \in F(\bar{x})$ and $S$ is closed, then

$$
\bar{x} \in S \text {-l-Mzer } \Longleftrightarrow \bar{x} \in \operatorname{st} \operatorname{argminP}(\bar{x}) .
$$

(ii) If $S$ is open, $S+S \subseteq S$ and $F(\bar{x})$ is compact, then

$$
\bar{x} \in S \text {-l-Mzer } \Longleftrightarrow \bar{x} \in \operatorname{argminP}(\bar{x}) .
$$

Remark 3.17. (i) Jiménez et al. ([18], Thm. 5.6) established Theorem 3.16(i) under the assumptions of $K$ closedness and $K$-properness.

(ii) It is known that $E_{(-\operatorname{int} K)}(A) \neq \emptyset$ for any compact set $A \subseteq Y$ (see [11], Rem. 2.5), where $K$ is closed convex pointed solid cone. However, $E_{S}(A)$ may be empty for a compact set with respect to an arbitrary set $S$. For instance, if $S=\left\{\left(y_{1}, y_{2}\right) \in \mathbb{R}^{2}: y_{2}>0\right\}$ and $A=[0,1] \times[0,1]$, then $E_{S}(A)=\emptyset$. Hence, the nonemptiness of $E_{S}(F(\bar{x}))$ in Theorem 3.16(ii) can not be relaxed even though $F(\bar{x})$ is compact.

We now present complete scalarizations of the set of $S$-l-minimizers.

Theorem 3.18. Let $0_{Y} \in \operatorname{cl} S, S+S \subseteq S$ and $E_{S}(F(x)) \neq \emptyset$, for every $x \in M$. Then, the following assertions hold:

(i) For each $x \in M \backslash\{\bar{x}\}$, if $\inf _{y \in F(x)} \Delta_{S}(y-\bar{y})$ is attained for every $\bar{y} \in F(\bar{x})$ and $S$ is closed, then

$$
S \text {-l-Mzer }=\bigcup_{u \in M} \text { st } \operatorname{argminP}(u) .
$$

(ii) If $S$ is open and $F$ is compact-valued on $M$, then

$$
S \text {-l-Mzer }=\bigcup_{u \in M} \operatorname{argminP}(u) .
$$

Proof. (i) From Theorem 3.16(i) we have $S$-l-Mzer $\subseteq \bigcup_{u \in M}$ st $\operatorname{argminP}(u)$. Let $\bar{x} \in$ st $\operatorname{argminP}(u)$ for some $u \in M$ and $x \in M \backslash\{\bar{x}\}$ be such that $F(x) \preceq_{S}^{l} F(\bar{x})$. Using Lemma 3.10(i) we get $\mathcal{D}(F(x), F(u)) \leq \mathcal{D}(F(\bar{x}), F(u))$ which contradicts the fact that $\bar{x} \in \operatorname{st} \operatorname{argminP}(u)$.

(ii) Proof follows on similar lines using Theorem 3.16(ii) and Lemma 3.10(ii).

We now give an example to illustrate the above theorem.

Example 3.19. Let $X=\mathbb{R}, Y=\mathbb{R}^{2}, M=[0,1]$. Define $F: X \rightrightarrows Y$ as

$$
F(x)= \begin{cases}\{(0,1)\}, & \text { if } x \leq 0 \\ \{(t, 0): t \in \mathbb{R}\}, & \text { if } 0<x<1 \\ \{(1,1)\}, & \text { if } x \geq 1\end{cases}
$$

If $S$ is as in Figure 1, then all the conditions of Theorem 3.18(i) hold and $S$-l-Mzer $=\{0,1\}$. The parametric scalar functions corresponding to the problem $(\mathrm{P}(0)),(\mathrm{P}(1))$ and $(\mathrm{P}(u))$ for $0<u<1$, respectively, are as follows:

$$
\mathcal{D}(F(x), F(0))= \begin{cases}0, & \text { if } x \leq 0 \\ 1, & \text { if } 0<x<1 \\ \frac{1}{\sqrt{2}}, & \text { if } x \geq 1\end{cases}
$$




$$
\begin{aligned}
& \mathcal{D}(F(x), F(1))= \begin{cases}\frac{1}{\sqrt{2}}, & \text { if } x \leq 0, \\
1, & \text { if } 0<x<1, \\
0, & \text { if } x \geq 1,\end{cases} \\
& \mathcal{D}(F(x), F(u))=0, \text { for all } x \in X .
\end{aligned}
$$

Therefore, st $\operatorname{argmin} \mathrm{P}(0)=\{0\}$, st $\operatorname{argminP}(1)=\{1\}$ and st $\operatorname{argmin} \mathrm{P}(u)=\emptyset$ for $0<u<1$ and hence, $S$-l-Mzer $=\bigcup_{u \in M}$ st $\operatorname{argminP}(u)$.

\section{Continuity of a SCAlar map ASsociated With oriented Distance maP}

Han and Huang [12] investigated continuity and convexity properties of an extended Gerstewitz function considered in [13]. Using these properties they established the lower and upper semicontinuity of the strong approximate solution map for a parametric set optimization problem. Inspired by their work, in this section we establish the continuity of the nonlinear scalar function $\mathcal{D}$ which leads to an existence result for $S$-l-minimizers. For this purpose we associate a map $\Theta: X \times X \rightarrow \mathbb{R} \cup\{ \pm \infty\}$ with the map $\mathcal{D}$, defined as

$$
\Theta(x, u):=\mathcal{D}(F(x), F(u)) .
$$

Theorem 4.1. If $F$ is continuous and compact-valued on $X$, then $\Theta$ is continuous on $X \times X$.

Proof. To establish the lower semicontinuity of $\Theta$, where $\Theta(x, u)=\sup _{v \in F(u)} \inf _{y \in F(x)} \Delta_{S}(y-v)$, we need to show that $L_{\alpha}(\Theta):=\{(x, u) \in X \times X: \Theta(x, u) \leq \alpha\}$ is closed for each $\alpha \in \mathbb{R}$. For a fixed $\alpha \in \mathbb{R}$, let $\left(\left(x_{n}, u_{n}\right)\right)_{n \in \mathbb{N}} \subseteq$ $L_{\alpha}(\Theta)$ be such that $\left(x_{n}, u_{n}\right) \rightarrow(\bar{x}, \bar{u})$. Let $\bar{v} \in F(\bar{u})$. Since $u_{n} \rightarrow \bar{u}$ and $F$ is lower semicontinuous at $\bar{u}$, there exists a sequence $\left(v_{n}\right)_{n \in \mathbb{N}}$ with $v_{n} \in F\left(u_{n}\right)$ for sufficiently large $n$ such that $v_{n} \rightarrow \bar{v}$. As $\Theta\left(x_{n}, u_{n}\right) \leq \alpha$ it follows that $\inf _{y \in F\left(x_{n}\right)} \Delta_{S}\left(y-v_{n}\right) \leq \alpha$, for each $n \in \mathbb{N}$. Now, $F\left(x_{n}\right)$ is compact so there exists $y_{n} \in F\left(x_{n}\right)$ such that

$$
\Delta_{S}\left(y_{n}-v_{n}\right) \leq \alpha
$$

for each $n \in \mathbb{N}$. Since $F$ is upper semicontinuous at $\bar{x}$ therefore by Lemma 2.2(i) there exist a subsequence $\left(y_{n_{k}}\right)_{k \in \mathbb{N}}$ of $\left(y_{n}\right)_{n \in \mathbb{N}}$ and $\bar{y} \in F(\bar{x})$ such that $y_{n_{k}} \rightarrow \bar{y}$. By Proposition 2.3(i) we know $\Delta_{S}$ is continuous and hence, from (4.1) we get

$$
\inf _{y \in F(\bar{x})} \Delta_{S}(y-\bar{v}) \leq \Delta_{S}(\bar{y}-\bar{v}) \leq \alpha .
$$

As the above inequality holds for any $\bar{v} \in F(\bar{u})$ it yields $\sup _{v \in F(\bar{u})} \inf _{y \in F(\bar{x})} \Delta_{S}(y-v) \leq \alpha$, that is, $(\bar{x}, \bar{u}) \in L_{\alpha}(\Theta)$.

We next prove the upper semicontinuity of the map $\Theta$, that is, the set $U_{\alpha}(\Theta):=$ $\{(x, u) \in X \times X: \Theta(x, u) \geq \alpha\}$ is closed for each $\alpha \in \mathbb{R}$. For a fixed $\alpha \in \mathbb{R}$, let $\left(\left(x_{n}, u_{n}\right)\right)_{n \in \mathbb{N}} \subseteq U_{\alpha}(\Theta)$ be such that $\left(x_{n}, u_{n}\right) \rightarrow(\bar{x}, \bar{u})$. Let $\bar{y} \in F(\bar{x})$. By the lower semicontinuity of $F$ at $\bar{x}$, there exists a sequence $\left(y_{n}\right)_{n \in \mathbb{N}}$ with $y_{n} \in F\left(x_{n}\right)$ for sufficiently large $n$ such that $y_{n} \rightarrow \bar{y}$. Since $\Theta\left(x_{n}, u_{n}\right) \geq \alpha$, $g(v)=\inf _{y \in F\left(x_{n}\right)} \Delta_{S}(y-v)$ is upper semicontinuous and $F$ is compact-valued it follows that there exists $v_{n} \in F\left(u_{n}\right)$ such that $\inf _{y \in F\left(x_{n}\right)} \Delta_{S}\left(y-v_{n}\right) \geq \alpha$, for each $n \in \mathbb{N}$. Hence, we have

$$
\Delta_{S}\left(y_{n}-v_{n}\right) \geq \alpha
$$

for each $n \in \mathbb{N}$. Again by the upper semicontinuity of $F$ at $\bar{u}$ there exist a subsequence $\left(v_{n_{k}}\right)_{k \in \mathbb{N}}$ of $\left(v_{n}\right)_{n \in \mathbb{N}}$ and $\bar{v} \in F(\bar{u})$ such that $v_{n_{k}} \rightarrow \bar{v}$. Thus from (4.2) we have $\Delta_{S}(\bar{y}-\bar{v}) \geq \alpha$ which further implies that $(\bar{x}, \bar{u}) \in U_{\alpha}(\Theta)$.

Remark 4.2. We observe that the above theorem extends Corollary 1 of [15] for $S=-K$, where $K$ is a proper closed convex cone. However, the proof follows on different lines using cone upper and lower semicontinuity notions.

The following is an outcome of the above theorem and establishes the continuity of the objective function of the parametric scalar problem. 
Corollary 4.3. If $F$ is continuous and compact-valued on $M$ and $u \in M$, then $\Theta(., u)$ is continuous on $M$.

In view of Corollary 4.3 it is clear that the problem $(\mathrm{P}(u))$ has a minimal solution if $M$ is compact. Hence using Theorem 3.18(ii) we have the following existence theorem for $S$-l-minimizers.

Theorem 4.4. Let $0_{Y} \in \operatorname{cl} S, S+S \subseteq S$ and $E_{S}(F(x)) \neq \emptyset$ for every $x \in M$. If $S$ is open, $M$ is compact and $F$ is continuous and compact-valued on $M$, then $S$-l-Mzer $\neq \emptyset$.

Remark 4.5. (i) In view of Remark 3.5, if $F$ is continuous and compact-valued on $M$ and $M$ is compact, then there exists a weak $l$-minimal solution of $(\mathrm{P})$.

(ii) As every $S$-l-minimizer is an $S$-l-minimal solution of $(\mathrm{P})$ therefore the above theorem provides an existence theorem for $S$-l-minimal solutions as well where the preference relation $\preceq_{S}^{l}$ need not be quasi order. However, the existence theorem of $S$-l-minimal solutions established in Theorem 5.1 of [24] is applicable only when $\preceq_{S}^{l}$ quasi order.

\section{StABility via SCALARIZATION}

In this section, we discuss the convergence of minimal solution sets by virtue of scalarization. By employing scalarization results and continuity of the map associated with oriented distance map, we establish the upper and lower Painlevé-Kuratowski set convergence of the sequence of minimal solution sets of parametric scalarized problems to the set of $S$-l-minimizers of $(\mathrm{P})$.

From [22] we recall the notion of Painlevé-Kuratowski convergence for a sequence of sets in $X$. The sequence $\left(\Omega_{n}\right)_{n \in \mathbb{N}} \subseteq X$ is said converge to the set $\Omega \subseteq X$ in the Painlevé-Kuratowski sense, if $\operatorname{Ls}\left(\Omega_{n}\right) \subseteq \Omega \subseteq \operatorname{Li}\left(\Omega_{n}\right)$, where

$$
\begin{aligned}
& \operatorname{Ls}\left(\Omega_{n}\right):=\left\{x \in X: \exists x_{n_{k}} \rightarrow x \text { with } x_{n_{k}} \in \Omega_{n_{k}}\right\}, \\
& \operatorname{Li}\left(\Omega_{n}\right):=\left\{x \in X: \exists x_{n} \rightarrow x \text { with } x_{n} \in \Omega_{n}, \text { for } n \text { sufficiently large }\right\} .
\end{aligned}
$$

The inclusion $\operatorname{Ls}\left(\Omega_{n}\right) \subseteq \Omega$ (resp. $\Omega \subseteq \operatorname{Li}\left(\Omega_{n}\right)$ ) is referred to as upper (resp. lower) part of Painlevé-Kuratowski convergence, denoted by $\Omega_{n} \stackrel{\mathrm{K}}{\rightarrow} \Omega$ (resp. $\Omega_{n} \stackrel{\mathrm{K}}{\rightarrow} \Omega$ ). Clearly, $\operatorname{Li}\left(\Omega_{n}\right) \subseteq \operatorname{Ls}\left(\Omega_{n}\right)$.

We next recall from [26] that a sequence $\left(\Omega_{n}\right)_{n \in \mathbb{N}}$ of subsets of $X$ upper converges to a set $\Omega \subseteq X$ in the Hausdorff sense, if $e\left(\Omega_{n}, \Omega\right) \rightarrow 0$, where $e\left(\Omega_{n}, \Omega\right):=\sup _{x \in \Omega_{n}} d(x, \Omega)$. We denote this convergence by $\Omega_{n} \stackrel{\mathrm{H}}{\rightarrow} \Omega$. From Corollary 2.1 in [21] it is known that if $\Omega$ is a closed set and $\Omega_{n} \stackrel{\mathrm{H}}{\rightarrow} \Omega$, then $\Omega_{n} \stackrel{\mathrm{K}}{\rightarrow} \Omega$.

The following lemma will be used in the sequel to establish the Painlevé-Kuratowski set convergence.

Lemma 5.1 ([1], Lem. 3.3). If $\Omega_{n} \stackrel{\mathrm{H}}{\rightarrow} \Omega$, where $\left(\Omega_{n}\right)_{n \in \mathbb{N}}$ is a sequence of nonempty subsets of $X$ and $\Omega$ is a nonempty compact set in $X$, then for any sequence $\left(x_{n}\right)_{n \in \mathbb{N}}$ with $x_{n} \in \Omega_{n}$ there exists a subsequence $\left(x_{n_{k}}\right)_{k \in \mathbb{N}}$ of $\left(x_{n}\right)_{n \in \mathbb{N}}$ and $x \in \Omega$ such that $x_{n_{k}} \rightarrow x$.

We consider the following family of parametric scalar optimization problems by perturbing the feasible set of the scalar problem $(\mathrm{P}(u))$. We consider the problem $\left(\mathrm{P}_{n}(u)\right)$ for each $n \in \mathbb{N}$, where $M_{n} \subseteq X$ is a nonempty set and $u \in M_{n}$, as follows

$$
\begin{array}{ll}
\left(\mathrm{P}_{n}(u)\right) \quad & \text { Minimize } \mathcal{D}(F(x), F(u)) \\
& \text { subject to } x \in M_{n} .
\end{array}
$$

We denote the set of minimal solutions of $\left(\mathrm{P}_{n}(u)\right)$ by $\operatorname{argminP} \mathrm{P}_{n}(u)$.

We now establish the lower part of convergence of the set of minimal solutions of the perturbed problem $\left(\mathrm{P}_{n}(u)\right)$ to the set of minimal solutions of $(\mathrm{P}(u))$. 
Theorem 5.2. If the following conditions hold:

(a) $M_{n} \stackrel{\mathrm{K}}{\rightarrow} M$,

(b) $F$ is continuous and compact-valued on $X$,

(c) $M_{n} \stackrel{\mathrm{H}}{M}$ and $M$ is compact,

then $\bigcup_{u \in M_{n}} \operatorname{argminP}_{n}(u) \stackrel{\mathrm{K}}{\rightarrow} \bigcup_{u \in M} \operatorname{st} \operatorname{argminP}(u)$.

Proof. Let $\bar{x} \in \bigcup_{u \in M}$ st $\operatorname{argminP}(u)$, which implies that $\bar{x} \in \operatorname{st} \operatorname{argminP}(\bar{u})$, for some $\bar{u} \in M$, that is,

$$
\mathcal{D}(F(\bar{x}), F(\bar{u}))<\mathcal{D}(F(x), F(\bar{u})),
$$

for all $x \in M$. Since $M_{n} \stackrel{\mathrm{K}}{\rightarrow} M$ there exist sequences $\left(\bar{x}_{n}\right)_{n \in \mathbb{N}}$ and $\left(\bar{u}_{n}\right)_{n \in \mathbb{N}}$ with $\bar{x}_{n}, \bar{u}_{n} \in M_{n}$ such that $\bar{x}_{n} \rightarrow \bar{x}$ and $\bar{u}_{n} \rightarrow \bar{u}$. We claim that for sufficiently large $n$

$$
\mathcal{D}\left(F\left(\bar{x}_{n}\right), F\left(\bar{u}_{n}\right)\right) \leq \mathcal{D}\left(F(x), F\left(\bar{u}_{n}\right)\right) .
$$

for all $x \in M_{n}$. On the contrary, let there exists a subsequence $\left(n_{k}\right)_{k \in \mathbb{N}}$ and $\hat{x}_{n_{k}} \in M_{n_{k}}$ such that

$$
\mathcal{D}\left(F\left(\hat{x}_{n_{k}}\right), F\left(\bar{u}_{n_{k}}\right)\right)<\mathcal{D}\left(F\left(\bar{x}_{n_{k}}\right), F\left(\bar{u}_{n_{k}}\right)\right) .
$$

Since $M_{n} \stackrel{\mathrm{H}}{\rightarrow} M$ and $M$ is compact, therefore by Lemma 5.1 there exists a subsequence $\left(\hat{x}_{n_{k_{l}}}\right)_{l \in \mathbb{N}}$ of $\left(\hat{x}_{n_{k}}\right)_{k \in \mathbb{N}}$ and $\hat{x} \in M$ such that $\hat{x}_{n_{k_{l}}} \rightarrow \hat{x}$. Taking limit along the subsequences in (5.2) and using Theorem 4.1 we have

$$
\mathcal{D}(F(\hat{x}), F(\bar{u})) \leq \mathcal{D}(F(\bar{x}), F(\bar{u}))
$$

which contradicts (5.1).

The next theorem establishes the upper part of convergence of the set of minimal solutions of the perturbed problem $\left(\mathrm{P}_{n}(u)\right)$ to the set of minimal solutions of $(\mathrm{P}(u))$.

Theorem 5.3. If the conditions (a)-(c) of Theorem 5.2 hold, then

$$
\bigcup_{u \in M_{n}} \operatorname{argminP}_{n}(u) \stackrel{\mathrm{K}}{\bigcup_{u \in M}} \operatorname{argminP}(u) .
$$

Proof. Let $\bar{x} \in \operatorname{Ls}\left(\bigcup_{u \in M_{n}} \operatorname{argminP}_{n}(u)\right)$. Then, there exist a subsequence $\left(\bar{x}_{n_{k}}\right)_{k \in \mathbb{N}}$ with $\bar{x}_{n_{k}} \in$ $\bigcup_{u \in M_{n_{k}}} \operatorname{argminP} n_{n_{k}}(u)$ such that $\bar{x}_{n_{k}} \rightarrow \bar{x}$. Let $\bar{x}_{n_{k}} \in \operatorname{argminP}_{n_{k}}\left(u_{n_{k}}\right)$ for some $u_{n_{k}} \in M_{n_{k}}$. As $u_{n_{k}} \in M_{n_{k}}$ and $M_{n_{k}} \stackrel{\mathrm{H}}{\longrightarrow} M$, hence by Lemma 5.1 there exists a subsequence $\left(u_{n_{k_{l}}}\right)_{l \in \mathbb{N}}$ of $\left(u_{n_{k}}\right)_{k \in \mathbb{N}}$ and $\bar{u} \in M$ such that $u_{n_{k_{l}}} \rightarrow \bar{u}$. It is sufficient to show that $\bar{x} \in \operatorname{argminP}(\bar{u})$. Let $x \in M$. As $M_{n} \stackrel{\mathrm{K}}{\rightarrow} M$ there exists a sequence $\left(x_{n}\right)_{n \in \mathbb{N}}$ with $x_{n} \in M_{n}$ such that $x_{n} \rightarrow x$. As $\bar{x}_{n_{k_{l}}} \in \operatorname{argminP}{ }_{n_{k_{l}}}\left(u_{n_{k_{l}}}\right)$ we have

$$
\mathcal{D}\left(F\left(\bar{x}_{n_{k_{l}}}\right), F\left(u_{n_{k_{l}}}\right)\right) \leq \mathcal{D}\left(F\left(x_{n_{k_{l}}}\right), F\left(u_{n_{k_{l}}}\right)\right) .
$$

By Theorem 4.1 it follows that $\mathcal{D}(F(\bar{x}), F(\bar{u})) \leq \mathcal{D}(F(x), F(\bar{u}))$ and hence, $\bar{x} \in \operatorname{argminP}(\bar{u})$.

Finally using Theorem 3.18, 5.2 and 5.3 we have the following conclusion.

Theorem 5.4. Let $0_{Y} \in \operatorname{cl} S$ and $S+S \subseteq S$. Let $E_{S}(F(u)) \neq \emptyset$ for each $u \in M$ and the conditions (a)-(c) of Theorem 5.2 hold. Then, the following assertions hold: 
(i) If $S$ is closed, then

$$
\bigcup_{u \in M_{n}} \operatorname{argminP}_{n}(u) \stackrel{\mathrm{K}}{\rightarrow} S \text {-l-Mzer. }
$$

(ii) If $S$ is open, then

$$
\bigcup_{u \in M_{n}} \operatorname{argminP}_{n}(u) \stackrel{\mathrm{K}}{\rightarrow} S-l-\text { Mzer. }
$$

Remark 5.5. From Remark 2.5 of [11], we have $E_{-K}(A)$ and $E_{-\operatorname{int} K}(A)$ are nonempty for any compact set $A \in \mathcal{P}(Y)$ and hence, the following holds immediately from the above theorem and Remark 3.5.

Corollary 5.6. Let $K$ be a closed convex pointed cone with nonempty interior. If the conditions (a)-(c) of Theorem 5.2 hold, then

(i) $\bigcup_{u \in M_{n}} \operatorname{argminP}_{n}(u) \stackrel{\mathrm{K}}{\rightarrow} l$-SMin.

(ii) $\bigcup_{u \in M_{n}} \operatorname{argminP}_{n}(u) \stackrel{\mathrm{K}}{\rightarrow} l$-WMzer.

It may be observed that if $F$ is a single-valued map, then the notion of $S$-l-minimizer reduces to the notion of $S$-minimal solution considered in [7]. We denote the set of $S$-minimal solutions of (P) by $S$-Min. The following corollary is an consequence of Theorem 5.4.

Corollary 5.7. Let $0_{Y} \in \operatorname{cl} S$ and $S+S \subseteq S$. If the conditions (a)-(c) of Theorem 5.2 hold, then

(i) $\bigcup_{u \in M_{n}} \operatorname{argminP}_{n}(u) \stackrel{\mathrm{K}}{\rightarrow} S$-Min, provided $S$ is closed.

(ii) $\bigcup_{u \in M_{n}} \operatorname{argminP}_{n}(u) \stackrel{\mathrm{K}}{\longrightarrow} S$-Min, provided $S$ is open.

Remark 5.8. Shiva and Lalitha ([20], Thm. 5.6) gave a similar result for a unified notion of minimal solution using Gerstewitz scalarizing function.

\section{Conclusions}

This paper focused on the study of scalarization using an oriented distance function of sup-inf type and stability in Painlevé-Kuratowski convergence sense for a unified set optimization problem. A unified notion of minimal solutions is characterized in terms of minimal solutions of a parametric scalar problem with objective function as the generalized oriented distance function. Further, the continuity of a map associated with the generalized oriented distance function led to an existence result for the unified minimal solutions as well as for the minimal solutions considered in [24]. We further established the lower and upper part of PainlevéKuratowski convergence of the sequence of minimal solution sets of perturbed scalarized problems to the set of minimal solutions of the given set optimization problem. It would be interesting to investigate the convexity and other properties of the scalarizing function to explore existence results as well as convexity and connectedness of the minimal solution set.

Acknowledgements. The authors would like to thank the anonymous referees for their careful reading of the manuscript and for providing valuable comments and suggestions.

Disclosure statement. No potential conflict of interest was reported by the authors.

Funding. This research for the first author is supported by CSIR, Senior Research Fellowship, India, National R\&D Organization (Ack. No: 151012/2K18/1) and the second author is supported by MATRICS scheme of Department of Science and Technology, India. 


\section{REFERENCES}

[1] L.Q. Anh, T.Q. Duy, D.V. Hien, D. Kuroiwa and N. Petrot, Convergence of solutions to set optimization problems with the set less order relation. J. Optim. Theory Appl. 185 (2020) 416-432.

[2] C.R. Chen, S.J. Li and K.L. Teo, Solution semicontinuity of parametric generalized vector equilibrium problems. J. Global Optim. 45 (2009) 309-318.

[3] C.R. Chen, X. Zuo, F. Lu and S.J. Li, Vector equilibrium problems under improvement sets and linear scalarization with stability applications. Optim. Methods Softw. 31 (2016) 1240-1257.

[4] J. Chen, Q.H. Ansari and J.C. Yao, Characterizations of set order relations and constrained set optimization problems via oriented distance function. Optimization 66 (2017) 1741-1754.

[5] M. Dhingra and C.S. Lalitha, Approximate solutions and scalarization in set-valued optimization. Optimization 66 (2017) 1793-1805.

[6] F. Flores-Bazán and E. Hernández, A unified vector optimization problem: complete scalarizations and applications. Optimization 60 (2011) 1399-1419.

[7] F. Flores-Bazán, F. Flores-Bazán and S. Laengle, Characterizing efficiency on infinite-dimensional commodity spaces with ordering cones having possibly empty interior. J. Optim. Theory Appl. 164 (2015) 455-478.

[8] C. Gerth and P. Weidner, Nonconvex separation theorems and some applications in vector optimization. J. Optim. Theory Appl. 67 (1990) 297-320.

[9] C. Gutiérrez, E. Miglierina, E. Molho and V. Novo, Convergence of solutions of a set optimization problem in the image space. J. Optim. Theory Appl. 170 (2016) 358-371.

[10] T.X.D. Ha, A Hausdorff-type distance, a directional derivative of a set-valued map and applications in set optimization. Optimization 67 (2018) 1031-1050.

[11] Y. Han and N.J. Huang, Well-posedness and stability of solutions for set optimization problems. Optimization 66 (2017) 17-33.

[12] Y. Han and N.J. Huang, Continuity and convexity of a nonlinear scalarizing function in set optimization problems with applications. J. Optim. Theory Appl. 177 (2018) 679-695.

[13] E. Hernández and L. Rodríguez-Marín, Nonconvex scalarization in set optimization with set-valued maps. J. Math. Anal. Appl. 325 (2007) 1-18.

[14] J.B. Hiriart-Urruty, Tangent cones, generalized gradients and mathematical programming in Banach spaces. Math. Oper. Res. 4 (1979) 79-97.

[15] L. Huerga, B. Jiménez, V. Novo and A. Vílchez, Six set scalarizations based on the oriented distance: continuity, convexity and application to convex set optimization. Math. Methods Oper. Res. 93 (2021) 413-436.

[16] K. Ike, M. Liu, Y. Ogata and T. Tanaka, Semicontinuity of the composition of set-valued map and scalarization function for sets. J. Appl. Numer. Optim. 1 (2019) 267-276.

[17] B. Jiménez, V. Novo and A. Vílchez, A set scalarization function based on the oriented distance and relations with other set scalarizations. Optimization 67 (2018) 2091-2116.

[18] B. Jiménez, V. Novo and A. Vílchez, Characterization of set relations through extensions of the oriented distance. Math. Methods Oper. Res. 91 (2020) 89-115.

[19] B. Jiménez, V. Novo and A. Vílchez, Six set scalarizations based on the oriented distance: properties and application to set optimization. Optimization 69 (2020) 437-470.

[20] S. Kapoor and C.S. Lalitha, Stability and scalarization for a unified vector optimization problem. J. Optim. Theory Appl. 182 (2019) 1050-1067.

[21] Karuna and C.S. Lalitha, External and internal stability in set optimization. Optimization 68 (2019) $833-852$.

[22] A. Khan, C. Tammer and C. Zălinescu, Set-valued Optimization: An Introduction with Applications. Springer-Verlag, Berlin (2015).

[23] Khushboo and C.S. Lalitha, Scalarizations for a set optimization problem using generalized oriented distance function. Positivity 23 (2019) 1195-1213.

[24] Khushboo and C.S. Lalitha, A unified minimal solution in set optimization. J. Global Optim. $\mathbf{7 4}$ (2019) 195-211.

[25] E. Köbis and M.A. Köbis, The weighted set relation: characterizations in the convex case. J. Nonlinear Var. Anal. 5 (2021) 721-735.

[26] K. Kuratowski, Topology. Vol. II. New edition, revised and augmented. Translated from the French by A. Kirkor. Academic Press, New York-London; Państwowe Wydawnictwo Naukowe Polish Scientific Publishers, Warsaw (1968).

[27] I. Kuwano, Some minimax theorems of set-valued maps and their applications. Nonlinear Anal. 109 (2014) 85-102.

[28] I. Kuwano and T. Tanaka, Continuity of cone-convex functions. Optim. Lett. 6 (2012) 1847-1853.

[29] I. Kuwano, T. Tanaka and S. Yamada, Inherited properties of nonlinear scalarizing functions for set-valued maps. In: Nonlinear Analysis and Convex Analysis. Yokohama Publ., Yokohama (2010) 161-177.

[30] C.S. Lalitha and P. Chatterjee, Stability and scalarization of weak efficient, efficient and Henig proper efficient sets using generalized quasiconvexities. J. Optim. Theory Appl. 155 (2012) 941-961.

[31] G.M. Lee, D.S. Kim, B.S. Lee and N.D. Yen, Vector variational inequality as a tool for studying vector optimization problems. Nonlinear Anal. 34 (1998) 745-765. 
[32] J. Li and C. Tammer, Set optimization problems on ordered sets. Appl. Set-Valued Anal. Optim. 1 (2019) 77-94.

[33] P.-P. Liu, H.-Z. Wei, C.-R. Chen and S.-J. Li, Continuity of solutions for parametric set optimization problems via scalarization methods. J. Oper. Res. Soc. Chin. 9 (2021) 79-97.

[34] A.M. Rubinov and R.N. Gasimov, Scalarization and nonlinear scalar duality for vector optimization with preferences that are not necessarily a pre-order relation. J. Global Optim. 29 (2004) 455-477.

[35] Y.D. Xu and S.J. Li, A new nonlinear scalarization function and applications. Optimization 65 (2016) $207-231$.

[36] A. Zaffaroni, Degrees of efficiency and degrees of minimality. SIAM J. Control Optim. 42 (2003) 1071-1086.

[37] W.Y. Zhang, S.J. Li and K.L. Teo, Well-posedness for set optimization problems. Nonlinear Anal. 71 (2009) $3769-3778$.

\section{Subscribe to Open (S2O) A fair and sustainable open access model}

This journal is currently published in open access under a Subscribe-to-Open model (S2O). S2O is a transformative model that aims to move subscription journals to open access. Open access is the free, immediate, online availability of research articles combined with the rights to use these articles fully in the digital environment. We are thankful to our subscribers and sponsors for making it possible to publish this journal in open access, free of charge for authors.

\section{Please help to maintain this journal in open access!}

Check that your library subscribes to the journal, or make a personal donation to the $\mathrm{S} 2 \mathrm{O}$ programme, by contacting subscribers@edpsciences.org

More information, including a list of sponsors and a financial transparency report, available at: https://www. edpsciences.org/en/maths-s2o-programme 